\title{
Mixoma e Infarto agudo de miocardio: ¿Hallazgo incidental o agente causal?
}

\author{
Emiliano Gustavo Spampinato \\ Leandro Satulovsky \\ Luis Guillermo Godoy- Zammarrelli \\ Cristina Margarita Lanfranco, \\ María Laura Plastino
}

\section{Correspondencia}

Cristina Margarita Lanfranco

cris.lanfranco@gmail.com

Unidad de Eco Doppler Cardiovascular. Área de Cardiología. Hospital Italiano de La Plata, Buenos Aires, Argentina

Recibido: $12 / 04 / 2020$

Aceptado: $24 / 02 / 2021$

En línea: 30/04/2021

Citar como: Spampinato E, Satulovsky L, Godoy Zammarrelli L, Lanfranco C, Plastino M, Mixoma e infarto agudo de miocardio: Hallazgo incidental o agente causal?. Rev Ecocar Pract (RETIC). 2021 (Abril); 4 (1): 30-33. doi: 10.37615/retic.v4n1a11.

Cite this as: Spampinato E, Satulovsky L, Godoy Zammarrelli, Lanfranco, Plastino M, Myxoma and acute myocardial infarction: an incidental finding or causal agent? Rev Ecocar Pract (RETIC). 2021 (Abril); 4 (1): 30-33. doi: 10.37615/retic.v4n1a11.

\section{Palabras clave}

$\triangleright$ Infarto agudo de miocardio

$\triangleright$ Tumores cardíacos

$\triangleright$ Mixoma

\section{Keywords}

$\triangleright$ Acute myocardial infarction

$\triangleright$ Cardiac tumors

$\triangleright$ Myxoma

\section{RESUMEN}

Presentamos el caso de una paciente con síndrome coronario agudo con elevación del segmento ST tratada con fibrinolíticos y derivada para angioplastia de rescate. En el ecocardiograma transtorácico control (ETT), luego de la cinecoronariografía, se evidencia masa adherida al septum interauricular, sospechándose mixoma (MX) con diagnóstico diferencial de trombo en tránsito, por lo que se realiza ecocardiograma transesofágico (ETE), donde se visualiza la masa implantada en el septum interauricular con trombo adherido. El diagnóstico de mixoma se confirmó posteriormente durante la resección quirúrgica y anatomía patológica.

ABSTRACT
We present the case of a patient with acute coronary syndrome with ST segment elevation with fibrinolytic
treatment derived for rescue angioplasty. In the transthoracic echocardiogram (TTE), after the coronary angio-
graphy, a mass attached to the interatrial septum is observed, with suspicion of myxoma vs thrombus in transit.
Transesophageal echocardiogram (TEE) is performed, the mass is observed related to the interatrial septum with
thrombus attached. The diagnosis of myxoma was confirmed during surgical resection and pathological ana-
tomy.

\section{Presentación del caso}

Mujer de 42 años de edad, fumadora de 20 paquetes/año, con antecedentes heredo familiares positivos (hermano fallecido por infarto de miocardio a los 52 años), quien consulta a un hospital por dolor torácico opresivo de 20 minutos de duración. Le realizan electrocardiograma (Figura 1) donde se evidencia elevación del segmento ST de cara inferior. Además del dolor torácico, no presentaba signos de falla cardiaca aguda y estaba hemodinámicamente compensada. Recibe tratamiento trombolítico a los 40 minutos del inicio del dolor, persistiendo con el dolor a los 90 minutos pese a un descenso del segmento ST mayor al 50\%.

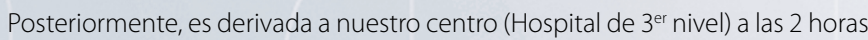
de haber culminado el fibrinolítico. Se presenta asintomática y con criterios de reperfusión positivos, por lo que se decide realizar cinecoronariografía (CCG) que muestra lesión leve en tercio medio de la arteria coronaria derecha (ACD) y oclusión del tercio distal del ramo postero-ventricular (Vídeo 1), sin otras lesiones significativas. Por tratarse de un vaso de fino calibre con lesión distal, se decide no realizar angioplastia.

Se solicita ecocardiograma Doppler (Vídeos 2 y 3) que muestra hipocinesia de los segmentos basales del septum inferior y pared inferior, con fracción de eyección del ventrículo izquierdo estimada en 63\%. Adicionalmente, se visualiza una masa eco-heterogénea dentro de la aurícula izquierda, con movimiento propio, adherida al septum interauricular cercana a de la fosa oval, con medida de1,6 x 0,8 cm y área de $1 \mathrm{~cm}^{2}$ (Vídeo 4, Figura 2), planteándose los diagnósticos de mixoma auricular versus trombo en tránsito. Por el último planteamiento, se realiza ecografía Doppler venosa de miembros inferiores que descarta trombosis venosa profunda.

Para mejor valoración de la masa auricular, se decide realizar un ecocardiograma transesofágico (ETE, Vídeos 5-7), visualizándose una masa heterogénea, vascularizada, con base de implantación amplia y fija en el septum interauricular a nivel de la fosa oval. Se constató, en su superficie libre, una imagen móvil, de distinta ecogenicidad, sugestiva de trombo adherido. 
Ante los hallazgos descritos, se anticoagula y se programa resección quirúrgica diferida, que se realiza luego de 4 meses, extrayéndose una pieza compatible con tumor cardiaco benigno tipo mixoma (Figura 3), confirmado posteriormente por anatomía patológica.

\section{Estudio por imagen}

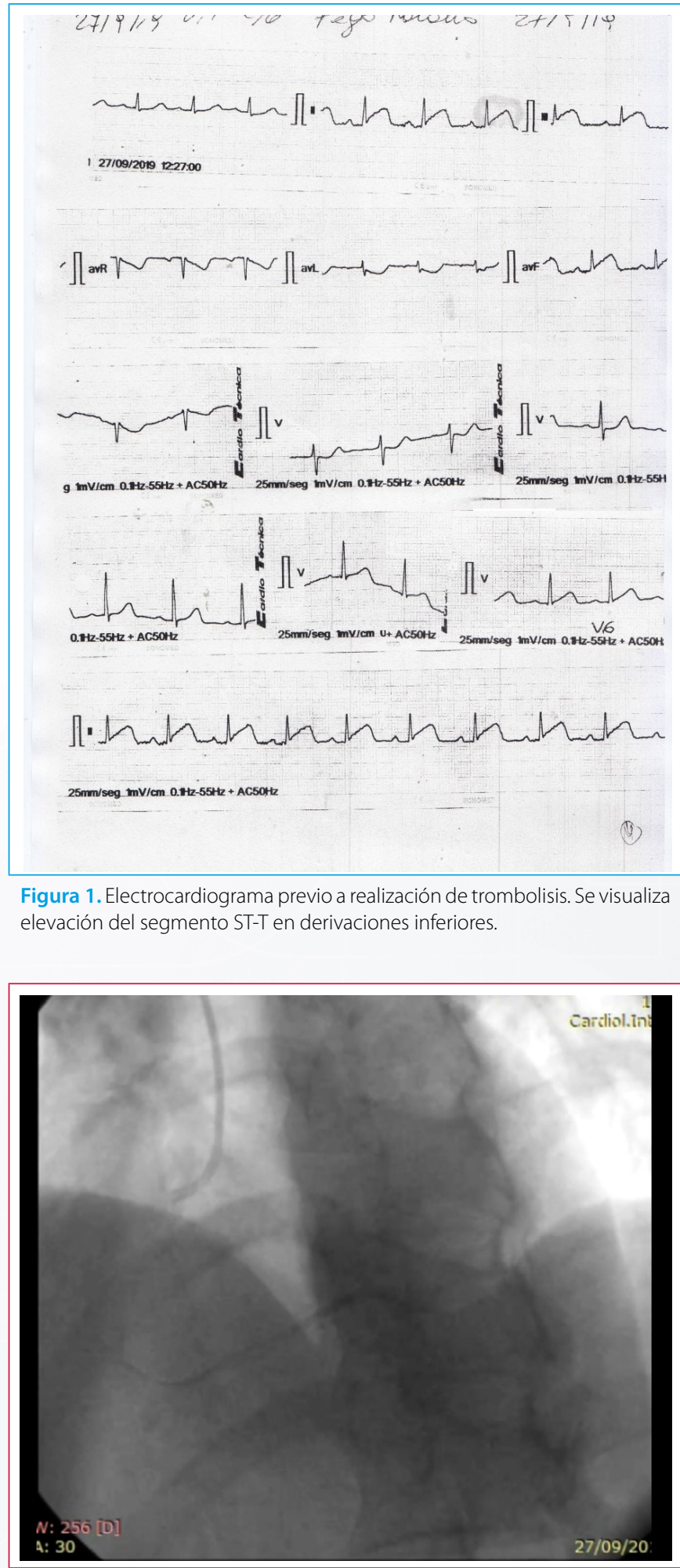

Vídeo 1. Cinecoronariografía, proyección oblicua anterior izquierda craneal, que evidencia la arteria coronaria derecha

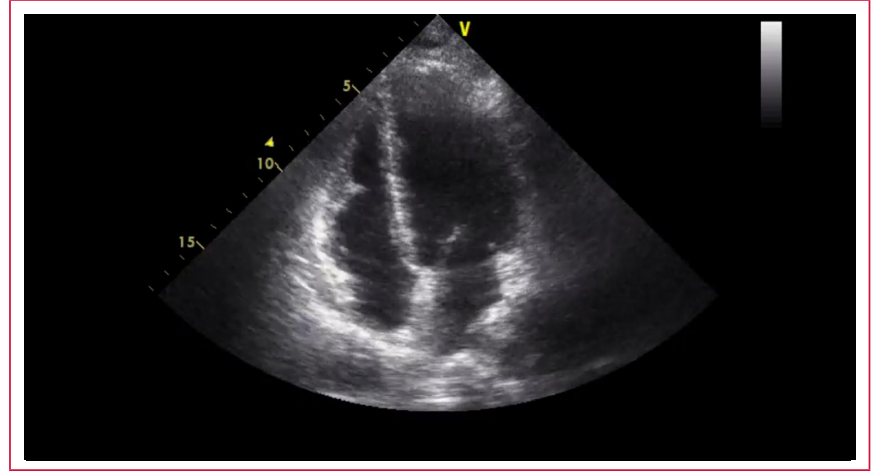

Vídeo 2. Ecocardiograma transtorácico, vista apical de 4 cámaras, donde se observa hipocinesia del segmento basal del septum inferior del ventrículo izquierdo

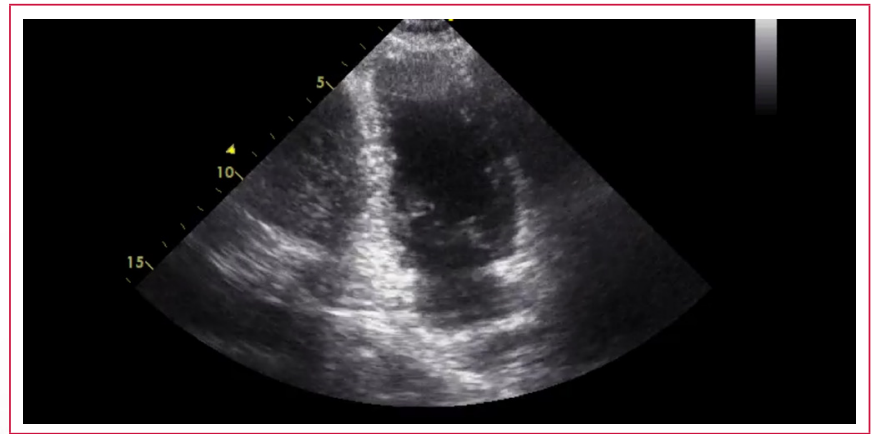

Vídeo 3. Ecocardiograma transtorácico, vista apical de 2 cámaras, donde se observa hipocinesia del segmento basal de pared inferior del ventrículo izquierdo

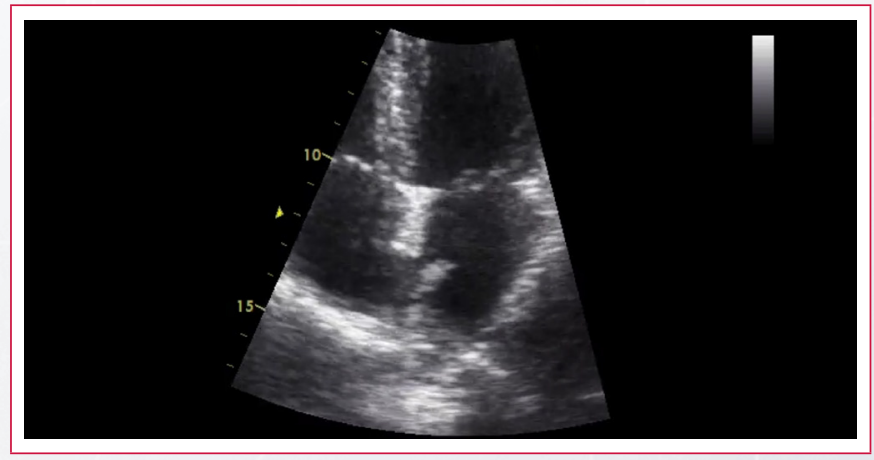

Vídeo 4. Ecocardiograma transtorácico, vista apical de 4 cámaras, con zoom en aurícula izquierda, observándose la masa adherida al tabique interauricular.

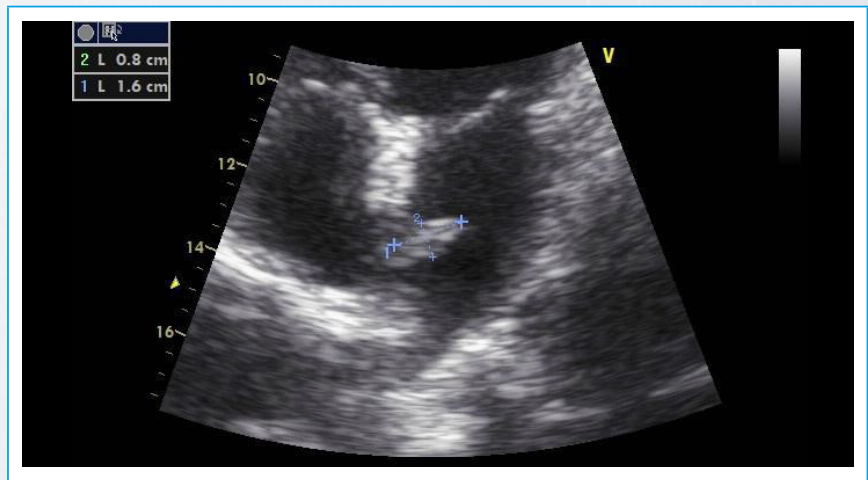

Figura 2. Ecocardiograma transtorácico, vista apical de 4 cámaras, con zoom a nivel del septum interauricular. Se observa masa heterogénea interauricular izquierda de $1,6 \times 0,8 \mathrm{~cm}$. 


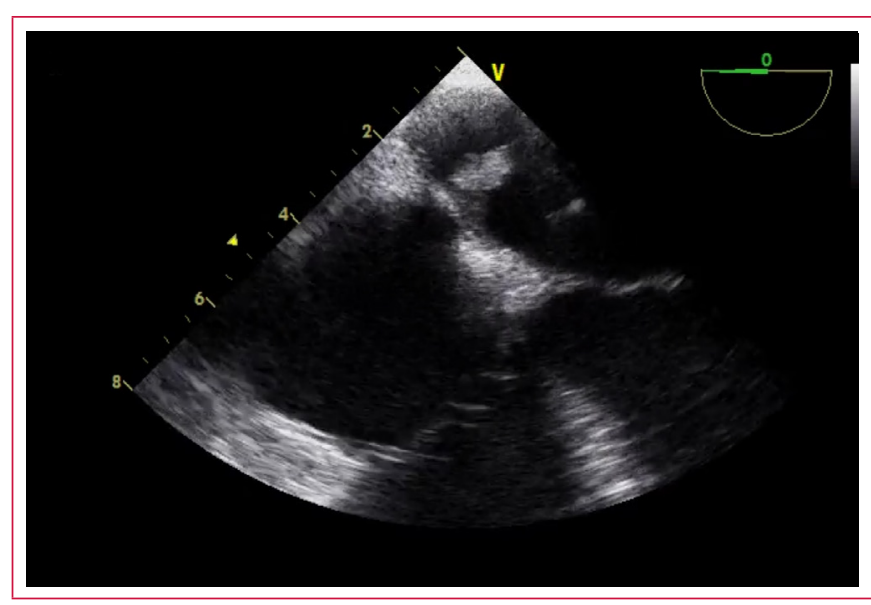

Vídeo 5. Ecocardiograma transesofágico, vista medio-esofágica 4 cámaras en $0^{\circ}$, donde se observa la masa de aspecto gelatinoso en aurícula izquierda. adherida al tabique interauricular

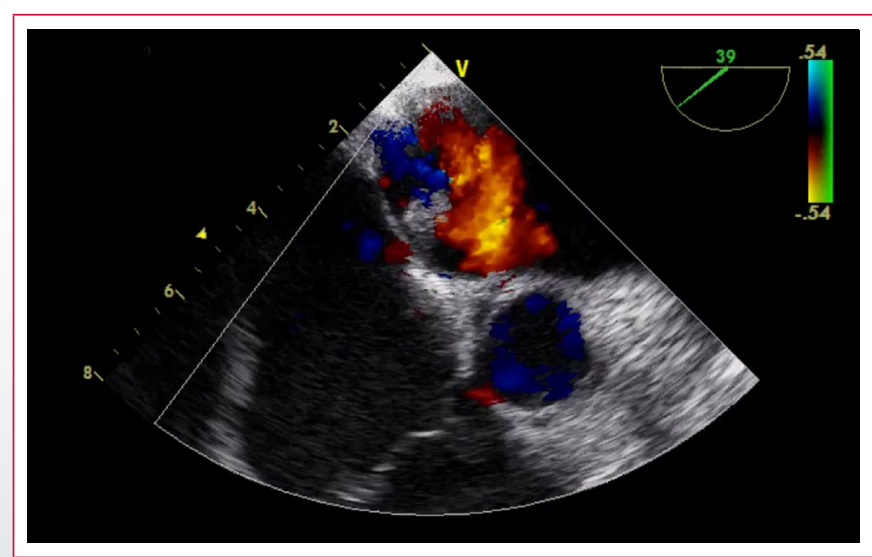

Vídeo 6. Ecocardiograma transesofágico con Doppler color, vista intermedia entre esófago alto-medio, donde se observa el septum interauricular anteriorizado, con la masa adherida al mismo

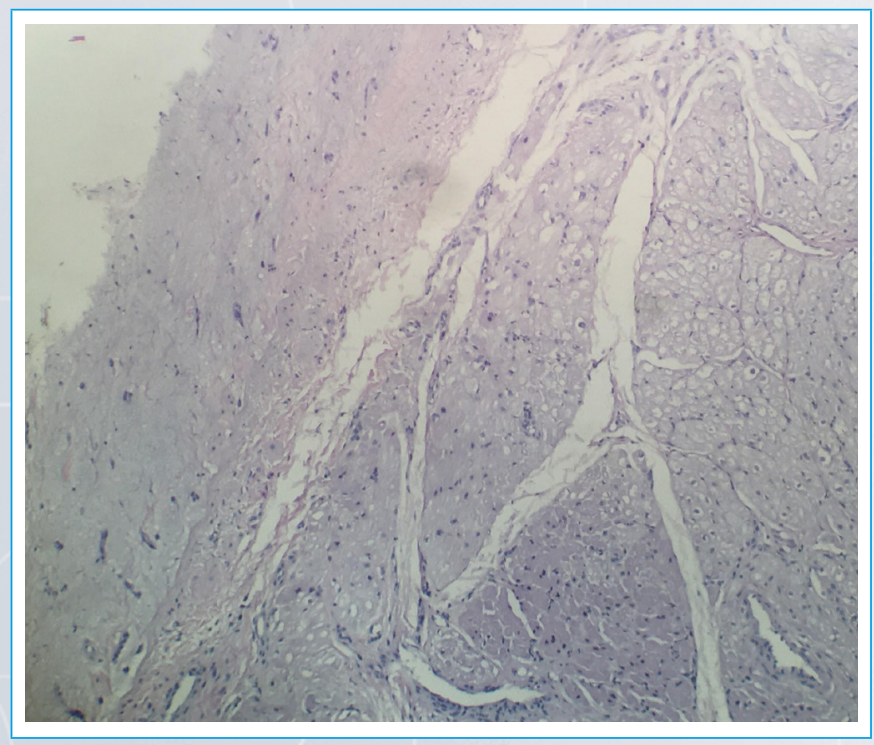

Figura 3. Anatomía histo-patológica de la pieza quirúrgica, donde se observa tejido muscular cardiaco con neoformación constituida por estroma mixoide y presencia de células poligonales y estrelladas, aisladas y en pequeños cordones y canales vasculares, sin atipias. Diagnóstico histológico de mixoma

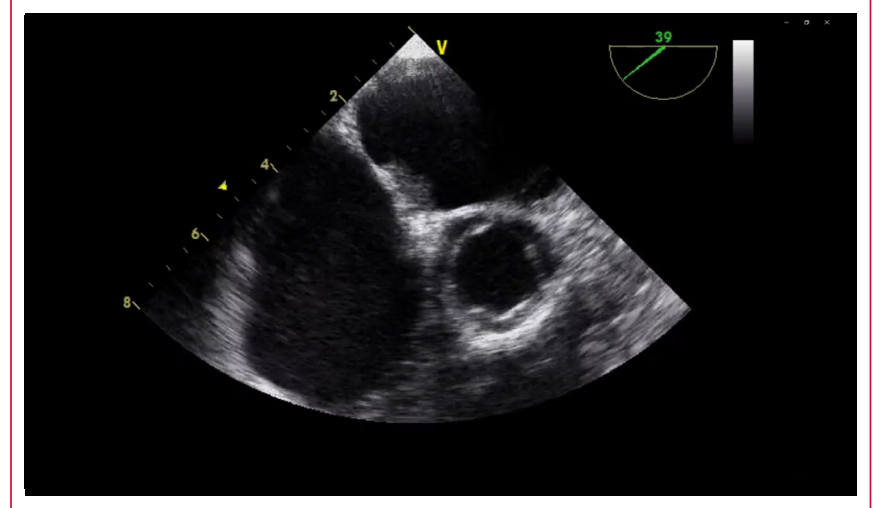

Vídeo 7. Ecocardiograma transesofágico con Doppler color, vista intermedia entre esófago alto-medio, donde se observa el septum interauricular anteriorizado, con la masa adherida al mismo

\section{Discusión}

Hemos presentado un caso donde se muestra la asociación de síndrome coronario agudo y mixoma, un hecho poco frecuente, aunque de importante impacto clínico y pronóstico.

Dentro de los tumores cardíacos primarios, los cuales presentan una incidencia baja, de alrededor $0.2 \%$, el mixoma es el tipo más común; llegando a representar entre el $50 \%$ al $85 \%$. No obstante, a pesar de considerarse benigno, desde el punto de vista histopatológico, su forma de presentación puede ser agresiva ${ }^{(1)}$. Generalmente son masas gelatinosas, fragmentables, pediculadas $y$ solitarias (94\%), con edad de presentación entre los 30-60 años (media de 50), más frecuente en mujeres, y localizándose mayormente en la aurícula izquierda. Su presentación es variada, desde asintomáticos hasta casos con clínica de obstrucción valvular mitral o de eventos tromboembólicos. La producción de émbolos puede estar dada por la fragmentación de la masa tumoral, trombosis asociada al tumor o una combinación entre estos factores ${ }^{(2)}$

Aunque la embolización sistémica es frecuente (30-60\% de los casos) ya que en su mayoría son tumores friables, la oclusión arterial coronaria es extremadamente infrecuente, ya que en sístole los ostium coronarios están protegidos por los velos de la válvula aórtica. No obstante, pueden ocurrir embolismos coronarios de material mixomatoso y ser causa de oclusión coronaria, posibilidad que debe considerarse sobre todo en pacientes jóvenes, sin factores de riesgo y con estudios de imágenes con arterias coronarias estructuralmente normales ${ }^{(3,4)}$.

Hay embolismos documentados a ambas arterias coronarias, principalmente de la derecha, que en dicho caso se asocia con infarto de pared inferior. Esta tendencia podría explicarse por el ángulo de la raíz aórtica con respecto a la horizontal y a la coronaria derecha que está situada caudalmente, por lo que los pequeños émbolos podrían ser llevados a ella más fácilmente que a la coronaria izquierda situada cefálicamente $e^{(5)}$.

La embolización coronaria puede limitarse a estas arterias o asociarse a embolismos múltiples, y ocurrir espontáneamente o secundario a manipulación quirúrgica(6).

En este contexto, el ecocardiograma es un pilar fundamental en el diagnóstico de las masas cardíacas, con sensibilidad y especificidad diagnósticas elevadas. Nos permite establecer la localización, tamaño, forma, sitio de implante y las características ecogénicas del tumor; elementos claves para descartar los 
posibles diagnósticos diferenciales, entre los que se encuentran trombos y vegetaciones $^{(7)}$.

En nuestro caso clínico, la paciente presentaba factores de riesgo para cardiopatía isquémica (tabaquismo y los antecedentes heredofamiliares), presentándose con dolor torácico y electrocardiograma con supradesnivel del ST inferior, trombolizándose y llevándose luego a CCG donde se constató oclusión del lecho distal del ramo postero-ventricular de la coronaria derecha, pese al tratamiento fibrinolítico realizado, culminando posteriormente con diagnóstico de mixoma auricular izquierdo evidenciado en la ecocardiografía control post CCG.

Con respeto al tratamiento del tumor, es eminentemente quirúrgico y suele ser definitivo; siendo su recidiva poco común (3\% en los tumores esporádicos y $22 \%$ en los tumores familiares), relacionada a su vez con la resección incompleta, por lo que se recomienda la realización de estudios ecocardiográficos periódicos en el seguimiento de estos pacientes ${ }^{(8)}$.

\section{Conclusión}

Frente a la sospecha de un síndrome coronario agudo en pacientes de mediana edad, con o sin factores de riesgo cardiovascular y sin lesiones epicárdicas significativas en el cateterismo cardíaco o con signos de embolia coronaria distal, la ecocardiografía resulta fundamental para la búsqueda de etiologías poco frecuentes como los tumores intracardiacos.

\section{Ideas para recordar}

- Los síndromes coronarios agudos asociados a mixomas son infrecuentes, pero existen y debe conocerse tal asociación por su impacto clínico y pronóstico.
- La sensibilidad diagnóstica de la ecocardiografía transtorácica y transesofágica para mixomas cardiacos es muy alta (93\% y 97\%; respectivamente), siendo las herramientas de primera elección para arribar al diagnóstico y guiar el tratamiento.

\section{Bibliografía}

1. Salas L, Tejera P, Pascucci D, Ausón V, Chacón L, Pizzorno A, et al. Mixoma auricular izquierdo en un paciente con embolia multiorgánica. Revista CONAREC 2019; 34 (149): 111-113.

2. Contreras A, Cragnolini A, Brenna E, Parisi G, Chamale R, Assante M, et al. Embolias múltiples causadas por mixoma auricular. Revista Facultad de Ciencias Médicas 2013; 70 (2): 91-93.

3. Kocaturk H, Karaman A, Bayram E, Colak MC, Yurtman V. Left atrial myxoma and concomitant atherosclerotic coronary artery disease. The Eurasian Journal of Medicine 2009; 41 (3): 202-204.

4. Haffner P, Monteiro V, Lins G, De Mattos E, De Andrade W, Villacorta H, et al. Infarto del miocardio y mixoma en aurícula izquierda. Arq Bras Cardiol:imagem cardiovasc. 2013;26 (4): 315-319.

5. Mohammad-Hassan N, Hamid-Reza RS, Ali-Reza S. The early presentation of atrial myxoma with acute myocardial infarction. Archives of Iranian Medicine, 2008; 11 (1): 98 - 102.

6. Calvellina J, Varela M. Infarto agudo miocárdico inferior secundario asociado a un mixoma de la aurícula izquierda An Med Asoc Med Hosp ABC 2000; 45 (3): 145-148

7. Ahumada-Zakzuk S, Ruiz-Pla F. Uso de ecocardiografía en la evaluación de masas cardíacas. Rev Colomb Cardiol. 2019; 26 (S1): 103-110.

8. S. Braun, Myocardial infarction as complication of left atrial myxoma, Int J Cardiol. 2005;101 (1): 115-21. 